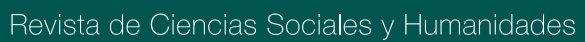

Diego Fabián Raza Carrillo diego.raza@uasb.edu.ec

Universidad Andina Simón Bolívar, Sede Ecuador.

Quito, Ecuador

ORCID:

https://orcid.org/0000-0003-3258-6344

\section{MEJORANDO LA COMUNICACIÓN EN LOS PROCESOS DE AUTOEVALUACIÓN DE LA CALIDAD EN UNIVERSIDADES}

IMPROVING COMMUNICATION IN SELF-EVALUATION PROCESSES OF QUALITY AT UNIVERSITIES

DOl:

https://doi.org/10.37135/chk.002.10.10 
Número 10 / ABRIL, 2020 (144-156)

\section{MEJORANDO LA COMUNICACIÓN EN LOS PROCESOS DE AUTOEVALUACIÓN DE LA CALIDAD EN UNIVERSIDADES}

IMPROVING
COMMUNICATION IN
SELF-EVALUATION
PROCESSES
OF QUALITY AT
UNIVERSITIES

\section{Resumen}

El objetivo de este artículo de reflexión es identificar problemas de comunicación en ejercicios de autoevaluación universitaria y proponer acciones para superarlos. La metodología utilizada fue de corte cualitativo a través de la observación directa de la comunicación en los procesos de autoevaluación en dos universidades ecuatorianas. A partir de identificar los problemas principales que, según la literatura, se presentan en el ejercicio comunicacional, se contrasta la experiencia observada en las dos universidades consideradas para el estudio, y a partir de ello se plantean recomendaciones para mejorar la comunicación y por tanto los procesos de autoevaluación de calidad universitaria. El resultado de este estudio sugiere que en la autoevaluación universitaria se presentan algunos problemas de comunicación tales como: utilización de modelos lineales de comunicación de una sola vía, inexacta codificación de mensajes, uso de eufemismos, prácticas defensivas, de aislamiento y ensimismamiento por parte de evaluadores y evaluados. A partir de estos resultados se sugieren acciones que según la teoría podrían aportar a la superación de estos problemas. El estudio tiene importancia, pues frente a los procesos de autoevaluación de la calidad de la educación superior, resulta necesario identificar elementos que inciden en el proceso, y la manera de gestionarlos.

Palabras clave: Comunicación, universidades, evaluación de calidad, autoevaluación, acreditación.

\begin{abstract}
The objective of this reflection paper is to identify communication problems in university self-evaluation procedures and suggest actions to overcome them. The methodology used had a qualitative nature through the direct observation of communication in self-assessment processes in two Ecuadorian universities. From the identification of the main problems that, according to the literature, are presented in the communicative process, the experience observed at these two universities considered for this study is contrasted, and thus, recommendations are established to improve communication along with the processes of university quality self-assessment. The result of this study suggests that there are some communication problems in university self-evaluation such as: the use of one-way linear communication models, inaccurate message coding, use of euphemisms, defensive practices, isolation and self-absorption by evaluators and evaluated parts. From these results, actions that according to the theory could contribute to overcoming these problems are suggested. The importance of the study lies on the need to identify elements that influence university self-evaluation processes, and the way to handle them out.
\end{abstract}

Keywords: Communication, universities, quality assessment, selfassessment, accreditation. 


\section{INTRODUCCIÓN}

Resulta común que las dificultades de interacción personal conlleven impactos en el desempeño esperado de los equipos, en el logro de sus objetivos, y en la construcción de espacios saludables de trabajo.

En efecto, existe evidencia del impacto de la comunicación en la organización y desempeño de grupos de trabajo (Guetzkow \& Simon 1955), en la relación del individuo con su entorno (Davison 1959), en la posibilidad de integrar efectivamente los esfuerzos de una organización (Murray 1965), en su relación con el clima organizacional y la satisfacción laboral (Muchinsky 1977), en la productividad en general (Hellweg \& Phillips 1982), en la innovación dentro de la organización (Monge, Cozzens \& Contractor 1992), en la identificación del personal con la organización y su prestigio externo (Smidts, Pruyn \& Riel 2001), en la generación de clima de confianza dentro de las organizaciones (Becerra \& Gupta 2003), en la mejor convergencia o comprensión mutua entre niveles directivos (Johnson \& Lederer 2005), en el nivel de cooperación entre individuos de la organización (Harbring 2006), en el desempeño de las personas en la administración pública (Garnett, Marlowe \& Pandey 2008), en el mejoramiento de la gestión de sus proveedores (Joshi 2009), en el uso de conocimiento para mejorar las capacidades organizacionales (Garicano \& Wu 2012), entre muchos otros impactos estudiados y documentados.

Los procesos de autoevaluación universitaria, igual que todo ejercicio interpersonal que se lleva a cabo en las organizaciones, requieren de la construcción de espacios y mecanismos de interacción efectiva entre las personas que intervienen en dichos ejercicios, justamente para regular estos efectos.

El hecho de que estos ejercicios de autoevaluación contienen un importante componente de control y ejercicio de poder, los hace particularmente propensos a revelar de manera clara problemas de interacción personal por roles que asume la parte evaluadora y evaluada, por espacios y dinámicas de poder y exigencia de cuentas, que convierten a estos ejercicios (en algunos casos) en momentos de estrés institucional que son potenciados muchas veces por prácticas comunicacionales imperfectas que, en lugar de aportar en la superación de las dificultades, las ahondan. Las mutuas posturas (tanto del evaluador como del evaluado) durante los procesos de autoevaluación, contribuyen a generar climas de tensión que dificultan el intercambio de información y por tanto complican innecesariamente el proceso evaluativo.

El objetivo de este estudio es identificar qué problemas se presentan en la comunicación durante los ejercicios de autoevaluación de la calidad universitaria, y proponer acciones que permitan palearlos.

\section{METODOLOGÍA}

Este trabajo es un artículo de reflexión que se origina en la participación del autor en el Curso Avanzado de Evaluación Universitaria, llevado a cabo en la Universidad Andina Simón Bolívar, sede Ecuador, entre octubre de 2018 y mayo de 2019, en Quito; y en su participación en los ejercicios de autoevaluación de calidad institucional en dos universidades ecuatorianas.

Se ha utilizado un método cualitativo, alineado a una posición paradigmática de constructivismo social, en el sentido de buscar comprender la complejidad del fenómeno estudiado (el proceso de comunicación durante ejercicios de autoevaluación universitaria), a partir del estudio de dos casos de autoevaluación en los que participó el autor, y utilizando la observación directa de dichos procesos en los que se prestó especial atención a la presencia de malas prácticas comunicacionales, que se fueron registrando para este estudio. Los sujetos 
observados fueron los personeros de las dos universidades que atendieron la visita de autoevaluación en las distintas dependencias institucionales, y los pares docentes que conformaron el equipo que condujo el ejercicio de autoevaluación.

Finalmente, a través de una revisión bibliográfica de lo que sugiere la literatura especializada, se identificaron posibles acciones para mejorar la comunicación en estos procesos.

\section{MARCO DE REFERENCIA}

\section{LA EVALUACIÓN Y AUTOEVALUACIÓN EN LA EDUCACIÓN SUPERIOR}

En el último siglo se han operado varios cambios estructurales en América Latina que han dado lugar a cambios en el sistema de educación superior que ha transitado por tres reformas importantes, según Rama (2006), de un modelo con énfasis en la autonomía y cogobierno, con una lógica pública y de lucha política, a un segundo modelo con una lógica privada, con un carácter más mercantilizado y marcadamente neoliberal (en la década de los 80), y a un tercer modelo (el actual) que está signado por la internacionalización de la educación superior que ha desatado una lógica nacional defensiva que, a su vez, ha impulsado el fortalecimiento de sistemas nacionales de evaluación y aseguramiento de la calidad de la educación superior.

Estos sistemas nacionales de aseguramiento de la calidad en la educación superior empiezan a consolidarse en la década de los 90 en distintos países de la región, con marcos normativos en los que tuvo alto impacto un proceso de negociación entre el Estado y las universidades, escuelas politécnicas e instituciones de educación superior en general, signado por tres características: 1. exagerado énfasis en la evaluación y autoevaluación como (única) estrategia de mejoramiento de la educación superior, 2. insuficiente (re)conocimiento de la (auto)evaluación como campo de conocimiento que devino en propuestas sobre todo empiristas, $\mathrm{y}$
3. heterogeneidad de las prácticas evaluativas en las instituciones del sistema de educación superior (Araujo 2014:61).

La evaluación de la educación superior debe comprenderse como un proceso político, en tanto (re) crea espacios de conflicto al ser un mecanismo de regulación y control en el que por tanto debe reflexionarse sobre por qué y para qué evaluar, quién debe evaluar y cómo se debe evaluar. Este proceso político, como mecanismo de regulación y control, ha generado lo que algunos pueden considerar una excesiva instrumentación burocrática que, a través de la determinación de indicadores y cifras, busca dar un resultado que pretende ser neutral y objetivo respecto a las condiciones de la institución de educación superior evaluada, y para lo cual es preponderante la participación de pares académicos en este proceso (Marquina 2008:9).

La evaluación puede ser realizada por evaluadores externos o internos. Cuando los evaluadores son internos, se trata de ejercicios denominados de evaluación institucional o autoevaluación, en los que su participación tiene como ventaja principal su conocimiento profundo de la organización a la que evalúan, si bien como desventaja se reconoce el riesgo de una posición menos objetiva al evaluar su propio espacio de trabajo en cuanto al cumplimiento de metas institucionales, uso de recursos, identificación de fortalezas y debilidades, situación en comparación con otras instituciones de educación superior, planteamiento de soluciones a problemas académicos y administrativos (Borroto \& Salas 2004).

De igual forma, se puede pensar en dos enfoques de evaluación (Miranda 2007), el enfoque diagnóstico-prescriptivo que se realiza casi exclusivamente a través de evaluaciones externas y que busca identificar nada más el cumplimiento de unos requisitos mínimos para lograr su autonomía; y el enfoque de autorregulación y mejora que está basado sobre todo en evaluaciones y mecanismos de seguimiento y mejoramiento internos y de carácter participativo, conducentes a identificar reflexivamente, elementos de la gestión universitaria que deben reforzarse, mejorar o eliminarse, con el fin de lograr un mejor desempeño. 
La autoevaluación institucional se constituye en un constructo complejo de relaciones entre diferentes personas de la institución, cada una muy diferente de la otra: personas de distinta jerarquía, con distintos roles en la institución, con distintas atribuciones; y todas ellas interactuando y por tanto cumpliendo un rol en la construcción de redes de información, y conectando/creando a la vez, redes de ideas, sucesos y prácticas dentro de la Universidad (Cardoso 2006).

\section{EVALUACIÓN Y AUTOEVALUACIÓN DE LA EDUCACIÓN SUPERIOR EN ECUADOR}

En Ecuador, tardíamente se crea el Consejo de Evaluación y Acreditación de la Educación Superior en el año 2000, y se realiza un primer ejercicio sistemático de evaluación de la educación superior a propósito del Mandato Constituyente 14, cuyo informe entregado en el 2009 da cuenta del análisis de alrededor de 70 universidades y escuelas politécnicas, y cerca de 300 institutos de educación post bachillerato en todo el país (CONEA 2009).

El modelo de evaluación aplicado en esa ocasión ha ido evolucionando: en un inicio consideró cuatro criterios (academia, estudiantes y entorno de aprendizaje, investigación, y gestión); para la siguiente evaluación en el año 2012 cambió a partir de una reorganización de sus elementos e indicadores, reforzando un énfasis cuantitativo y de categorización, con construcción de funciones de utilidad y un marcado enfoque economicista que permitía compensaciones de falencias en algunos aspectos evaluados con sobrecumplimiento en otros (Raza 2019:16), e instrumentando una visión productivista de la universidad en la que lo único importante era el puntaje acumulado para lograr mejor categorización (Villavicencio 2017).

Frente a la necesidad de realizar una nueva evaluación de la educación superior que se llevará a cabo a fines de 2019, se ha elaborado un nuevo modelo de evaluación que busca asegurar que las universidades y escuelas politécnicas en Ecuador cumplan, con unos mínimos requeridos por el Estado, alrededor de cuatro criterios: docencia, investigación, vinculación con la colectividad y condiciones institucionales. Este modelo responde a un Sistema Interinstitucional de Aseguramiento de la Calidad en la educación superior, que se sustenta principalmente en la autoevaluación y que busca evaluar las condiciones necesarias para la acreditación y ya no para la categorización de las universidades y escuelas politécnicas, a través de un conjunto más compacto de indicadores mayormente cualitativos (Consejo de Aseguramiento de la Calidad de la Educación Superior 2019:25), y en línea con la idea subyacente de que el Estado determina un conjunto de condiciones que construyen la idea social de lo que es Universidad, y que al ser evaluadas permiten o no el reconocimiento de la institución evaluada como tal (Labraña 2018:844).

Los procesos de acreditación estatal han desatado en las universidades y escuelas politécnicas -en general-, un conjunto de cambios relacionados con su gestión: cambios en misión, visión, normativa interna, mecanismos de control y seguimiento, cambios en la estructura en la que se empieza a considerar la existencia de oficinas de análisis institucional (o evaluación o planificación o similares), entre muchos otros (Lemaitre, Maturana, Zenteno \& Alvarado 2012), y si bien han revelado la necesidad de mejorar los procesos de planificación y evaluación (López 2007), también es cierto que han generado resultados positivos en la capacidad institucional de "acelerar procesos de mejoramiento de la calidad educativa" (De Vincenzi 2013).

Por ello parece importante evaluar también, además de las condiciones estructurales de la organización frente a los ejercicios de evaluación y autoevaluación, las condiciones más particulares de relacionamiento personal y comunicación durante estos ejercicios. 


\section{CONCEPTUALIZACIÓN DE LA COMUNICACIÓN}

Desde una postura crítica, se plantea que el capitalismo tardío ha traído cambios en distintas esferas del conocimiento y de la realidad: intensificación de las inequidades, degradación de la democracia, deterioro ambiental, enquistamiento de la racionalidad tecnocrática, entre otros fenómenos, cuyas causas se buscan en el avance tecnológico rampante, la liberalización a ultranza del capital, la ideologización del mercado como única medida y rasero, las políticas gubernamentales que fortalecen en lugar de regular estos fenómenos, así como el debilitamiento de la organización laboral. El conjunto de estos fenómenos adopta distintos nombres: posfordismo, neoliberalismo, economía de la información, neocolonialismo, posverdad, capitalismo comunicacional, entre otras designaciones que tratan de resumir (sin éxito) la variada y compleja trama de fenómenos socioeconómicos que perfilan la realidad actual, y en la que conviene preguntarse qué es la comunicación en las organizaciones en estos días y qué relación tiene con estos problemas generalizados que vive actualmente la sociedad (Kuhn, Ashcraft \& Cooren 2019).

Es necesario que la comunicación organizacional, si se plantea contribuir a la solución de los problemas actuales del mundo, se replantee a sí misma ontológicamente (Kuhn, Ashcraft \& Cooren 2017), mutando de una perspectiva de simple transmisión de mensajes a entenderse como mecanismo de relacionamiento, enlace y conexión entre las personas que emiten y reciben el mensaje, y de aceptación de que así las personas en sí mismas pueden ser consideradas como canal, instrumento y código a través del cual se expresa la realidad, planteándose su posibilidad de des/re/configurarla.

En este sentido, a la comunicación se puede definir como un proceso de creación de significado principalmente, en el que de manera operativa y ya instrumental se entiende que un emisor envía un mensaje a un receptor a través de un canal, utilizando un código, y buscando retroalimentación, según lo plantean Adler, Du Pré y Rodman (2018), quienes además alertan que el mensaje puede ser verbal y no verbal, planificado y no planificado (se puede comunicar sin intención), que es codificado (puesto en palabras por el emisor) no siempre de manera adecuada, y decodificado (extraído el supuesto contenido por parte del receptor, igual no siempre de forma adecuada).

\section{LA IMPORTANCIA DE LA COMUNICACIÓN}

La importancia de las soft skills o habilidades blandas en general, ha sido revelada en varios estudios a nivel mundial, en los que se muestra su necesidad de fortalecimiento en las organizaciones con el fin de mejorar el desempeño y resultados, hacer más factible el logro de objetivos, contribuir a mejores espacios laborales, entre otras ventajas.

El estudio de Rao (2018:215), por ejemplo, explora las habilidades blandas en organizaciones globales y en instituciones educativas, y expone lo que significan, su importancia, y la manera de adquirirlas. Diferencia a estas habilidades blandas de las denominadas duras, y propone un modelo de entrenamiento para su desarrollo. Se plantea como eminente la transmutación de una economía del conocimiento hacia una economía del autoconocimiento, y enfatiza la importancia del desarrollo de estas habilidades blandas, especialmente frente a la posibilidad cada vez más cierta de procesos productivos (y ejecutivos) dominados por inteligencia artificial, en empleados de todo rango: desde conserjes hasta altos ejecutivos, para lo cual incluso plantea un modelo de medición de habilidades blandas a modo de instrumento de diagnóstico previo antes de la selección de personal en una organización.

Esta importancia de las habilidades blandas, se muestra también en el estudio de Pedrosa, Feitosa, Dai Prá Martens y Ferreira da Silva (2018), en el que a través de un caso de estudio en Europa, se destaca que las organizaciones que gestionan proyectos de carácter mundial, obtienen mejores resultados cuando sus directores tienen más altos niveles de habilidades blandas, lo cual se revela en una correlación positiva encontrada entre estas 
dos variables, y que termina sugiriendo la necesidad de incluir a las habilidades blandas como requisito en la contratación de personas.

Otro estudio (Nadziakiewicz 2016), identifica de manera puntual la importancia de las habilidades blandas en ejercicios de auditoría, evaluación o control, al plantear que las habilidades blandas del auditor o evaluador (sea interno o externo), juegan un papel importantísimo en el éxito del ejercicio de evaluación, con lo cual concluye que las organizaciones para lograr mejores resultados, deberían entrenar a sus personeros para el desarrollo de este tipo de habilidades.

En consideración de que una adecuada comunicación es parte de las habilidades blandas que se requieren en una organización (Madrigal 2009), varios estudios se han llevado a cabo para mostrar la importancia de una buena comunicación en la organización.

El estudio de Romanescu (2016:29), por ejemplo, señala el rol fundamental de la comunicación en todas las funciones de una organización, enfatizando en el hecho de que debe ser considerada como un medio y no como un fin en sí misma, ya que permite una mejor ejecución de las distintas funciones en la organización.

Lewis (2019) complementa esta afirmación indicando que una adecuada comunicación tanto formal como informal es especialmente importante en procesos de cambio en una organización, como catalizador de los sentimientos de resistencia al cambio, e incertidumbre que acompañan normalmente los procesos de formación, transformación y evaluación en las organizaciones.

Shimizu (2017) expone la importancia de la comunicación por parte de los directivos en la exitosa implementación de la estrategia, junto con Moorcroft (2003) que de manera particular plantea su importante impacto en la distribución de recursos y consecuente logro de metas organizacionales.

Las universidades, frente a los ya dichos procesos de autoevaluación, no pueden ser la excepción en el reconocimiento de la necesidad organizacional de fortalecer las capacidades de comunicación de quienes trabajan en/con ella.

\section{PROBLEMAS PRESENTES EN EL PROCESO DE COMUNICACIÓN SEGÚN LA TEORÍA}

El estudio clásico de Melcher y Beller (1967), sugiere que uno de los principales problemas en la comunicación organizacional es la deficiente selección de canales y métodos de comunicación, derivada de varias condiciones que pueden estar presentes y que pueden ser incorrectamente identificadas en el proceso de comunicación.

Estas condiciones se refieren a: 1. la naturaleza de la comunicación, que está determinada por: el tipo de comunicación (dar una orden, solicitar o entregar información, llegar a consensos de actuación), la legitimidad de la misma (que dependerá de la autoridad o jerarquía de la persona que pide la información, busca consensos o pretende dar una orden), y los recursos que se requieren para llevar a cabo la comunicación (de tiempo, financieros o materiales); 2. las características de los distintos actores involucrados en la comunicación, es decir, de los receptores, transmisores, intermediarios, y otros implicados en el proceso, en cuanto a: su orientación hacia fines o medios (que determinará en última instancia el mayor o menor apego a seguir reglas y procedimientos preestablecidos), su confiabilidad en cuanto a generar certeza de que los intervinientes actuarán aportando positivamente a la comunicación (aclarando la información, generando un clima de confianza, escuchando atentamente), y sus capacidades de lenguaje que cuando son limitadas pueden afectar el contenido del mensaje; 3. la integración del sistema social, referida al tipo de relaciones interpersonales y patrones de interacción existentes entre los actores y grupos de actores involucrados en la organización; 4. las características requeridas por el proceso de comunicación para el canal, que tienen que ver con: la necesidad de rapidez en la transmisión y recepción del mensaje, la necesidad de contar con retroalimentación de manera oportuna, el interés de que se difunda de manera más amplia o focalizada el mensaje dentro de la organización, o de 
que se cuente con mayor o menor formalidad (y por tanto aceptación) especialmente en comunicaciones entre niveles jerárquicos, el costo de comunicar el mensaje por un canal u otro, entre otras.

Una incorrecta identificación del carácter de alguna de estas condiciones lleva entonces a una inadecuada selección del canal de transmisión del mensaje.

Otra investigación de referencia, la de Eisenberg y Witten (1987) apunta al nivel de claridad o ambigüedad en los procesos de comunicación, como posible fuente de dificultades, e identifica cuatro determinantes a tener en cuenta: contingencias individuales (referidas a los motivos, preferencias y estilos personales que determinan la manera en la que cada quien se comunica), contingencias relacionales (referidas al nivel de cercanía personal entre los miembros de la organización), contingencias organizacionales (el nivel de divulgación permitido por la organización para cierta información), y contingencias del entorno (condiciones externas a la organización que le obligan a guardar con cierta privacidad cierta información sensible).

Estos problemas que se dan en el ejercicio de comunicación, más otros que se refieren en distintos estudios, se pueden resumir en los siguientes: utilizar modelos lineales de una sola vía (pretendiendo que la comunicación fluya solo en un sentido), elegir mal los canales o hacer una mala de/re/codificación (Adler et al. 2018).

Estos riesgos y malas prácticas, sumados a problemas que pueden darse en el entorno, específicamente en las expectativas sociales que se pueden generar por parte del evaluador como del evaluado, y a los ruidos comunicacionales existentes (distracciones psicológicas especialmente producto del temor al proceso de evaluación) pueden resultar en tropiezos que interrumpan la normal marcha del ejercicio.

El uso de eufemismos (palabras inexactas e indirectas que se usan para no usar otras más directas y menos placenteras), las contradicciones entre el mensaje verbal y el no verbal, la falta de anticipación y consideración a las consecuencias que pueden acarrear los mensajes y la forma en la que se los transmite, son problemas comunicacionales comunes en las organizaciones.

De otra parte, son reconocidos los problemas en la escucha que se pueden presentar, y que dificultan una comunicación fluida (Adler et al. 2018), manifestándose de distintas maneras: el escuchador defensivo (percibe los comentarios de la otra parte como ataques), el escuchador emboscador (escucha atentamente para usar la propia información de la otra parte para atacarlo), el escuchador aislado (que ignora información que no es deseable para él), el escuchador insensible (falla en reconocer pensamientos o sentimientos que no son directamente expresados por la otra parte, y solo se centra en las palabras), el escuchador ensimismado (más preocupado de probar sus puntos de vista que de entender el de otros).

Estas conductas deben superarse si se quiere lograr fidelidad en la escucha, entendida como la congruencia entre lo que el receptor entiende y el emisor quiso comunicar.

\section{RESULTADOS Y DISCUSIÓN}

Durante las visitas realizadas a las dos universidades en las que se participó como parte del equipo de autoevaluación de la calidad, se observaron durante las entrevistas y durante la entrega de resultados, algunas prácticas inadecuadas de entre aquellas que se prevén en la literatura especializada, y que se resumen a continuación.

Utilización de modelos lineales de una sola vía: en las visitas se detectó esta mala práctica tanto en evaluadores como en evaluados. En el caso de los evaluados, se observaron exposiciones excesivamente largas con información que no era de interés para los evaluadores y que por tanto no aportaron en nada al ejercicio de evaluación y alargaron innecesariamente la visita generando un clima de incomodidad para ambas partes. Para el caso de los evaluadores, se detectó en otras visitas proble- 
mas similares con sujetos que monopolizaron el uso de la palabra, se extendieron innecesariamente en explicaciones y pedidos y que no permitían hablar ni al resto de integrantes del equipo evaluador ni a las personas de la institución evaluada. De igual forma, esta práctica conllevó malestar para ambas partes, y complicó sin necesidad el proceso de evaluación.

Inexacta codificación de los mensajes: tanto por parte de los evaluadores como de los evaluados. Si bien las personas que participaron como evaluadores y como evaluados pertenecen a la academia y comparten un conocimiento que se supone común respecto a la terminología relacionada con el proceso de evaluación de calidad universitaria, se observaron dificultades para codificar los mensajes tanto de pedido de información como de entrega de la misma, lo cual determinó la necesidad de aclaraciones, repreguntas, pedidos ampliados de información de manera innecesaria, entre otras dificultades generadas.

Uso de eufemismos: igual que en los problemas anteriores, esta dificultad también se presenta tanto en evaluadores como en evaluados. Durante las entrevistas realizadas, pero especialmente en la reunión de cierre del ejercicio en ambas instituciones, se evidenció el uso de eufemismos por parte de lado y lado de los equipos de evaluadores y evaluados. Es posible que, por un exceso de consideración entre pares académicos, la comunicación se vio viciada de términos ambiguos e inexactos, especialmente cuando se debía expresar desacuerdo. En lugar de ser directos en la comunicación y asertivos en los términos (aunque resulten incómodos) se prefería una comunicación imprecisa, difusa, con términos tibios y solo sugerentes en lugar de concretos y rotundos. Esto por supuesto determinó igualmente una dificultad en la comunicación que por tanto fue inadecuada en el ejercicio.

Escuchadores defensivos, aislados y ensimismados: durante las entrevistas se observó a varios evaluadores y evaluados exhibir conductas defensivas, de aislamiento y ensimismamiento, que tal como se definen en la literatura especializada, consideraban ataques personales algunas de las consultas o respuestas que se le daba (escuchador defensivo), ignoraba solicitudes o explicaciones que se le ofrecía si no le resultaban convenientes (escuchador aislado), y que era evidente que en lugar de un diálogo mantenía un intercambio de evidencias en la que solo buscaba demostrar sus puntos de vista sin interés en comprender el del otro (escuchador ensimismado).

Durante el ejercicio también se observaron buenas prácticas comunicacionales de gran cantidad de actores, y si se refieren solo las negativas no es porque no existan las positivas o porque sean mayor en número las negativas, sino porque es el propósito de este artículo referir las oportunidades de mejora para la comunicación en estos ejercicios de autoevaluación de la calidad universitaria.

Los resultados coinciden con lo planteado en la teoría como problemas principales de los procesos de comunicación, y si bien algunos de los problemas que plantea la teoría no se pudieron identificar (escuchador insensible, escuchador emboscador, y problemas de distracciones en el entorno), en general lo detectado se corresponde con lo que se plantea en la teoría como principales dificultades en el proceso de comunicación (Adler et al. 2018).

\section{RECOMENDACIONES PARA MEJORAR LA COMUNICACIÓN}

\section{LO SUGERIDO DESDE LA TEORÍA}

Adler, Du Pré y Rodman (2013; 2018) proponen algunas recomendaciones para mejorar la comunicación en las organizaciones.

Los autores plantean como fundamental la adopción de modelos de comunicación que superen lo lineal y le apuesten a lo transaccional, privilegiando una concepción de doble vía en la que envío y recepción ocurran de manera simultánea y de lado y lado. En este modelo transaccional, además, se debería pensar siempre en fortalecer el carácter interpersonal de la comunicación, entendiéndose aquello como una comprensión mutua de que las partes son individuos únicos con pensamientos $\mathrm{y}$ emociones propias, así como con marcos de refe- 
rencia de la realidad distintos.

En cuanto al tamaño de los grupos de trabajo (que inevitablemente se generan en los ejercicios de evaluación), sugieren privilegiar grupos pequeños en los que todos sus integrantes tengan la oportunidad de expresar su concepciones, anhelos, expectativas y visiones, participando así activamente en el proceso.

Para la actitud de escucha por parte de emisores y receptores, recomiendan adoptar diferentes estilos: escucha relacional (busca principalmente la construcción de cercanía emocional con la otra parte), escucha analítica (busca principalmente entender completamente el mensaje antes de hacer cualquier evaluación), escucha crítica (busca evaluar la calidad y precisión de lo que dice la otra parte), escucha de apoyo (busca mostrar soporte a otros que buscan ayuda en dilemas personales y profesionales). Esto como contrapeso a estilos negativos de escucha que fueron referidos más arriba.

Se propone también una práctica permanente de automonitoreo de las partes involucradas en la comunicación, de tal forma que se preste continua atención al comportamiento propio, buscando modelarlo sobre la marcha de tal forma que contribuya a una mejor escucha y comunicación en general, evitando dañar el autoconcepto y autoestima que tiene la otra parte, mostrando empatía en cada momento y sorteando la tentación fácil de caer en estereotipos y generalizaciones.

La aplicación de estas recomendaciones buscaría lograr seres competentes comunicacionalmente, entendiéndose esto como ser capaces de mantener la relación en términos aceptables para todas las partes (Adler et al. 2018), y lograr un balance entre efectividad en la comunicación e idoneidad en la misma (Spitzberg \& Cupach 2012), considerando la efectividad como el logro de lo que se propone en la situación particular en la que se lleva a cabo la comunicación: ¿se logró obtener toda la información que se requirió en el proceso de autoevaluación? ¿se logró que el equipo de contraparte prepare un informe más detallado que facilite la autoevaluación?; y comprendida la idoneidad, como el cumplimiento de las expectativas sociales que se generan en el proceso de comunicación: ¿se fue asertivo o impreciso, la interacción fue clara y directa o vaga y elusiva, se expresaron con objetividad las necesidades o se utilizó un tono pasivo-agresivo?

\section{CÓMO LOGRAR ESTO EN LA PRÁCTICA}

Vistos los problemas presentes en la comunicación durante ejercicios de autoevaluación, y que coinciden con los señalados por Adler et. al (2018) y se relatan en el acápite de Resultados y Discusión, parece necesario formar a evaluadores y evaluados en habilidades comunicacionales que les permitan sortear estos problemas.

Tal vez debería considerarse la construcción de un programa de formación para evaluadores (y también para evaluados), centrado en el desarrollo de habilidades blandas, particularmente en comunicación.

Si bien muchos cursos de comunicación hacen mucho énfasis en desarrollar competencias para la comunicación escrita, no debe descuidarse el entrenamiento en formas de comunicación verbal que se ha probado que tiene un excelente resultado en entornos organizacionales, en los que los sujetos pueden apreciar una verdadera transformación en sus habilidades comunicacionales (Yale 2014).

El programa de formación debería incluir los contenidos usuales y privilegiar metodologías constructivistas/participativas que favorezcan la praxis comunicacional en entornos de riesgo controlado, con el fin de preparar al evaluado/r para el proceso de interacción real en el que debe participar a propósito de la autoevaluación universitaria.

El programa debería entrenar a sus participantes en la escucha activa adecuada a cada situación, la selección idónea de canales y códigos, la proxémica como forma de usar el espacio en la comunicación, el uso correcto de paralenguaje (significados no lingüísticos de la expresión verbal: velocidad, tono, gravedad de las palabras), la cronémica (forma de usar y estructurar el tiempo durante la comu- 
nicación en los procesos de autoevaluación), entre otros contenidos sugeridos (Adler et al. 2013).

Se deberán además incluir otras competencias comunicacionales que se plantean hoy como indispensables: conducción de reuniones, gestión de equipos, manejo de conflictos, presentación de resultados, motivación a subordinados y escritura de propuestas y reportes (Hynes, Worley \& Dyrud 2002).

Todos estos contenidos, y otros que deberán seguirse construyendo, deberían siempre estar enfocados en lograr una mejor comunicación a largo plazo en las organizaciones en general y en las universidades en particular, para lo cual se debe buscar favorecer la comunicación de dos vías en todos los niveles de la organización, fortalecer la comunicación abierta y franca en los niveles de autoridad, y explorar continuamente las necesidades de todos los involucrados en los procesos de comunicación (Richmond 2011).

No se debe descartar el uso de plataformas tecnológicas de desarrollo de soft skills (de la comunicación particularmente) como ya se ha probado y sistematizado el uso de estas plataformas de manera exitosa (Theurelle-Stein \& Barth 2017).

\section{CONCLUSIONES}

Si bien la importancia de la comunicación en los resultados organizacionales ha sido abundantemente estudiada desde hace décadas, poco se ha indagado sobre el rol que cumple en los procesos de autoevaluación de la calidad en la educación superior. Del ejercicio realizado es evidente que los problemas comunes en la comunicación también se presentan en entornos universitarios, y que dificultan la delicada tarea de autoevaluación de la calidad. La literatura sugiere un conjunto de recomendaciones para mejorar la calidad, entre ellas la formación y capacitación en técnicas comunicacionales, lo cual se sugiere que también se implemente entre evaluadores y evaluados para mejorar la comunicación y por tanto la eficiencia en los ejercicios de autoevaluación de la calidad universitaria.

Estos procesos de capacitación deberían llevarse a cabo considerando una malla curricular completa y exhaustiva que (como ya se ha dicho) contemple contenidos para fortalecer las capacidades comunicacionales tanto verbales, escritas, y no-verbales, con metodologías constructivistas y altamente participativas que permitan a los participantes de estos procesos (evaluadores y evaluados), aprender practicando con el fin de que durante los ejercicios de autoevaluación de la calidad universitaria, el intercambio de información se lleve a cabo de manera más eficiente, contribuyendo así a la idoneidad del proceso y al logro de mejores resultados en el mismo.

\section{REFERENCIAS BIBLIOGRÁFICAS}

Adler, R., Du Pré, A. \& Rodman, G. (2013). Understanding Human Communication. Oxford, Inglaterra: Oxford University Press.

Adler, R., Du Pré, A. \& Rodman, G. (2018). Essential Communication. Oxford, Inglaterra: Oxford University Press.

Araujo, S. M. (2014). La evaluación y la Universidad en Argentina: Políticas, enfoques y prácticas. Revista de la educación superior, 43(172), 57-77.

Becerra, M. \& Gupta, A. K. (2003). Perceived Trustworthiness within the Organization: The Moderating Impact of Communication Frequency on Trustor and Trustee Effects. Organization Science, 14(1), 32-44.

Borroto Cruz, E. R. \& Salas Perea, R. S. (2004). Acreditación y evaluación universitarias. Educación Médica Superior, 18(3), 1-1.

Cardoso Nunes, L. (2006). As dimensões da auto-avaliação institucional: Tecendo redes de redes. Ensaio: Avaliação e Políticas Públicas em 
Educação, 14(52), 339-348. doi: 10.1590/ S0104-40362006000300004

CONEA. (2009). Evaluación de Desempeño Institucional de las Universidades y Escuelas Politécnicas del Ecuador. Quito: CONEA.

Consejo de Aseguramiento de la Calidad de la Educación Superior. (2019). Modelo de evaluación externa de universidades y escuelas politécnicas 2019. Quito: Consejo de Aseguramiento de la Calidad de la Educación Superior.

Davison, W. P. (1959). On the Effects of Communication. The Public Opinion Quarterly, 23(3), 343-360.

De Vincenzi, A. (2013). Evaluación institucional y mejoramiento de la calidad educativa en tres universidades privadas argentinas. Revista iberoamericana de educación superior, 4(9), 76-94.

Eisenberg, E. M. \& Witten, M. G. (1987). Reconsidering Openness in Organizational Communication. The Academy of Management Review, 12(3), 418-426. doi: 10.2307/258509

Garicano, L. \& Wu, Y. (2012). Knowledge, Communication, and Organizational Capabilities. Organization Science, 23(5), 1382-1397.

Garnett, J. L., Marlowe, J. \& Pandey, S. K. (2008). Penetrating the Performance Predicament: Communication as a Mediator or Moderator of Organizational Culture's Impact on Public Organizational Performance. Public Administration Review, 68(2), 266-281.

Guetzkow, H. \& Simon, H. A. (1955). The Impact of Certain Communication Nets upon Organization and Performance in Task-Oriented Groups. Management Science, 1(3/4), 233-250.

Harbring, C. (2006). The Effect of Communication in Incentive Systems: An Experimental Study. Managerial and Decision Economics, 27(5), 333-353.

Hellweg, S. A. \& Phillips, S. L. (1982). Communication and Productivity in Organizations. Public Productivity Review, 6(4), 276-288. doi: $10.2307 / 3380183$

Hynes, G. E., Worley, R. B. \& Dyrud, M.A. (2002). Strategies forTeaching Managerial Communication. Business Communication Quarterly, 65(3), 86-92. doi:10.1177/108056990206500311
Johnson, A. M. \& Lederer, A. L. (2005). The Effect of Communication Frequency and Channel Richness on the Convergence between Chief Executive and Chief Information Officers. Journal of Management Information Systems, 22(2), $227-252$.

Joshi, A. W. (2009). Continuous Supplier Performance Improvement: Effects of Collaborative Communication and Control. Journal of Marketing, 73(1), 133-150.

Kuhn, T., Ashcraft, K. \& Cooren, F. (2017). The Work of Communication: Relational Perspectives on Working and Organizing in Contemporary Capitalism. New York, NY: Routledge.

Kuhn, T., Ashcraft, K. \& Cooren, F. (2019). Introductory Essay: What Work Can Organizational Communication Do? Management Communication Quarterly, 33(1), 101-111. doi: $10.1177 / 0893318918809421$

Labraña Vargas, J. (2018). De qué hablamos cuando hablamos de universidades. El Estado Chileno y la idea contemporánea de la universidad. Avaliação: Revista da Avaliação da Educação Superior (Campinas), 23(3), 834-847. doi:10.1590/s1414-40772018000300015

Lemaitre, M. J., Maturana, M., Zenteno, E. \& Alvarado, A. (2012). Cambios en la gestión institucional en universidades, a partir de la implementación del Sistema Nacional de Aseguramiento de la Calidad: La experiencia chilena. Calidad en la educación, (36), 21-52. doi:10.4067/ S0718-45652012000100001

Lewis, L. (2019). Organizational Change: Creating Change Through Strategic Communication. Hoboken, NJ: Wiley-Blackwell.

López Leyva, S. (2007). Evaluación institucional y factores de cambio: La percepción de los académicos de tres universidades del noroeste de México. Revista de la educación superior, 36(144), 7-22.

Madrigal Torres, B. (2009). Habilidades directivas. México: McGraw Hill Interamericana.

Marquina, M. (2008). Académicos como pares evaluadores en el sistema argentino de evaluación de universidades: Diez años de experiencia. Revista de la educación superior, 37(148), 7-21.

Melcher, A. J. \& Beller, R. (1967). Toward a Theory of Organization Communication: Consid- 
eration in Channel Selection. The Academy of Management Journal, 10(1), 39-52. doi: $10.2307 / 255243$

Miranda J., C. (2007). Educación superior, mecanismos de aseguramieto de la calidad y formación docente: Un debate pendiente en Chile. Estudios pedagógicos (Valdivia), 33(1), 95-108. doi:10.4067/S0718-07052007000100006

Monge, P. R., Cozzens, M. D. \& Contractor, N. S. (1992). Communication and Motivational Predictors of the Dynamics of Organizational Innovation. Organization Science, 3(2), 250274.

Moorcroft, D. (2003). Linking communication strategy with organizational goals. Strategic Communication Management, 7(6), 24.

Muchinsky, P. M. (1977). Organizational Communication: Relationships to Organizational Climate and Job Satisfaction. The Academy of Management Journal, 20(4), 592-607. doi: $10.2307 / 255359$

Murray, W. (1965). Effective Communication as a Means of Integrating an Organization. Management International, 5(2/3), 185-200.

Nadziakiewicz, M. (2016). The Role of Soft Skills in Decision Making by Auditors. Rola Kompetencji Miękkich W Podejmowaniu Decyzji Przez Audytorów, (94), 151-159.

Pedrosa, N., Feitosa, D., Dai Prá Martens, C. \& Ferreira da Silva, L. (2018). Relevância dos soft skills em gerenciamento de projetos globais. Revista Gestão \& Tecnologia, 18(3), 245-260.

Rama, C. (2006). La tercera reforma de la educación superior en América Latina y el Caribe: Masificación, regulaciones e internacionalización. Revista Educación y Pedagogía, 18(46), 13-24.

Rao, M. S. (2018). Soft skills: Toward a sanctimonious discipline. On the Horizon, 26(3), 215-224. doi:10.1108/OTH-06-2017-0034

Raza, D. F. (2019). Evaluación y acreditación universitaria en Ecuador. Revista de Educación Superior en América Latina, O(6). Recuperado de http://rcientificas.uninorte.edu.co/index.php/ esal/article/viewFile/12183/214421443896

Richmond, R. (2011). Developing a sustainable leadership communication strategy. Strategic Communication Management, 15(8), 10.
Romanescu, M. L. (2016). The Importance of Managerial Communication in the Activity of a Firm. Annals of the University Dunarea de Jos of Galati: Fascicle: XVII, Medicine, 22(3), 29-33.

Shimizu, K. (2017). Senders' Bias: How Can Top Managers' Communication Improve or Not Improve Strategy Implementation? International Journal of Business Communication, 54(1), 52-69. doi:10.1177/2329488416675449

Smidts, A., Pruyn, A. Th. H. \& Riel, C. B. M. van. (2001). The Impact of Employee Communication and Perceived External Prestige on Organizational Identification. The Academy of Management Journal, 44(5), 1051-1062.

Spitzberg, B. H. \& Cupach, W. R. (2012). Handbook of Interpersonal Competence Research. New York, NY: Springer Science \& Business Media.

Theurelle-Stein, D. \& Barth, I. (2017). Les soft skills au coeur du portefeuille de compétences des managers de demain. Revue Management et Avenir, (95), 129-151. doi:10.3917/mav.095.0129

Villavicencio, A. (2017). Calidad, excelencia y evaluación en la universidad ecuatoriana. Quito: Paradiso Editores.

Yale, R. N. (2014). The Impromptu Gauntlet: An Experiential Strategy for Developing Lasting Communication Skills. Business \& Professional Communication Quarterly, 77(3), 281-296. doi:10.1177/2329490614537874 